\title{
An Innovative Transport Layer Protocol for Smart Grid Communications
}

\author{
Mohamed EL Brak \\ Abdelmalek Essaadi University \\ Morocco
}

\author{
Said EL Brak \\ Abdelmalek Essaadi University \\ Morocco
}

\author{
Mohamed Essaaidi \\ Mohammad V University \\ Morocco
}

\begin{abstract}
The communication technology for power systems a major focus in the development and deployment of smart grid, the SCTP protocol, which was initially designed for transporting signaling messages over IP networks, is now an established in different domain, and lately in Smart Grid communications. SCTP can provide high-performance transmission in both wired and wireless networks. This paper describes different components of smart grid and the smart grid communications layer then we propose a new approach to employ the Stream Control Transmission Protocol (SCTP) in a smart grid, by using the two very significant characteristics offered by SCTP multi-homing and multi-streaming respectively.

The simulation results show the comparison between two protocols of the transport layer, TCP and SCTP in terms of the throughput and delay. The simulation works have been conducted in NS2 network simulator.
\end{abstract}

\section{Keywords}

Smart Grid, TCP, SCTP, Multi-homing, Multi-streaming, NS2

\section{INTRODUCTION}

Integrating Information technologies in electrical grid system is the key concept of the Smart Grid. Traditionally, electrical grid system is the interconnections of the transmission system responsible of delivering the power from the power generation to the electrical distribution. However, the Smart grid term refer not only to the transmission system but also to the distribution system which delivers electricity from distribution substations to consumers. So, Smart grid is an electrical system that includes the intelligent part to the different operations of the traditional power grid as: "power generation, electricity transmission, electricity distribution, and electricity control and consumption\|” [2]

From the reliability point of view, this technology uses twoway flows of energy and information from the power generation to the consumer in order to create a widely distributed automated energy delivery network. Furthermore, the smart grid provides the ability to control and react to events and conditions from power stations to individual appliances, and also gather real time relevant measurement with the aim of decreasing the high costs of meeting peak demand and incorporating distributed renewable energies source.

Customers will play a big role in smart grid and they will interact with utility to manage peak power consumption through demand response protocol. This will benefit the utility in avoiding building costly energy generation. It will also benefit the customer as they will be able to benefit from low cost as well as the ability to make money through feed in tariff. This interaction will require two-way electricity and information communication among customers and utility through the Advanced Metering Infrastructure (AMI). Utilities will be able to communicate with the consumers, monitor and possibly control their power consumption [3]. These communications could be achieved through several layers with reference to the OSI reference model. The OSI model has seven layers namely, application, presentation, session, transport, network, data link and physical layers. In this paper, our focus is on the transport protocols which are part of the transport layer. Transport layer provides two types of service to an application. They are connection oriented and connection-less service. Connection oriented service provides reliable, full duplex connection ensuring end-to-end error detection and correction. While a connectionless service provides higher speeds as they do not provide flow control and error correction. The requirements for the transport protocols for Smart Grid are identified as: Secure, Reliable, High, Availability, Real Time and Scalable.

Few of the important transport protocols that find its place in Smart Grid are listed below.

- Transmission Control Protocol [TCP]

- Stream Control Transmission Protocol [SCTP]

- User Datagram Protocol [UDP]

- Wireless Datagram Protocol [WDP]

- $\quad$ Split TCP

- $\quad$ Cyclic UDP [CUDP]

- Wireless Profiled TCP [WP-TCP]

A proposal for the smart grid operators utilize SCTP as the principle transport protocol for their smart grid communications, by using the two very significant characteristics offered by SCTP multi-homing and multistreaming respectively [4].

This paper is organized as follows: Section 2 Smart grid conceptual model are presented. Section 3 presents Communication system for smart grid. Section 4 we proposed smart grid communication using SCTP and brief overviews. Section 5 and Section 6 we presented smart grid communication using multi-homing and multi-streaming SCTP respectively. Results and performance analysis, and comparisons are presented in section 7. Finally, conclusions is drawn in Section 8.

\section{SMART GRID CONCEPTUAL MODEL}

\subsection{The seven domains}

The conceptual model described by NIST (National Institute of Standards and Technology) supports the planning and organization of the interconnected networks that forms the 
Smart Grid. For this reason, NIST adopted the approach of dividing the Smart Grid as a High-Level framework that defines seven important domains. Each domain contains actors and applications. Actors include devices, systems or programs; for instance smart meters, solar generators, and control systems. Applications are the tasks performed by one or more actors within a domain, for instance home automation, solar energy generation and energy management etc.

The conceptual model is also described for the regulators to accomplish public policy goals with the business objectives. The main idea is to modernize the nation's electric power infrastructure and establishing a clean energy economy. Regulations, adopted by the FERC at the federal level and state level, govern many aspects of the Smart Grid. [5].

Naturally, the actors in a particular domain can interact with the actors in the other domains. The concepts for the Figure 1 are defined as the following:

Operations: Actors involved in the smooth operation of the power system.

Bulk generation: The generators of electricity in bulk quantities. May also store energy for later distribution.

Transmission: The carriers of bulk electricity over long distances. May also store and generate electricity.

Distribution: The distributors of electricity to and from customers. May also store and generate electricity.

Customer: The end users of electricity. May also generate, store, and manage the use of energy. Traditionally, three customer types are discussed, each with its own domain.

Markets: The markets are where grid assets are bought and sold. Actors in the Markets domain exchange price and balance supply and demand within the power system.

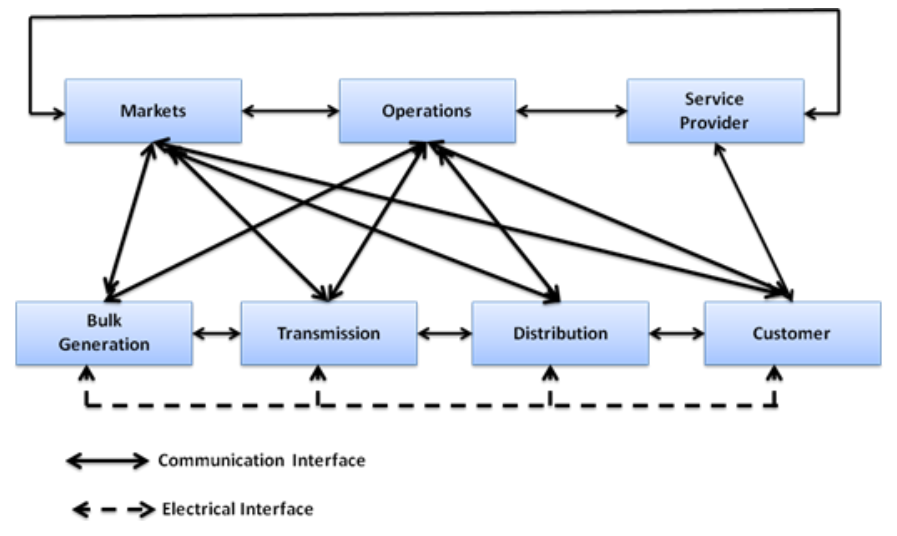

Figure 1 SG Conceptual Model

\section{COMMUNICATIONS SYSTEM FOR \\ SMART GRID}

Communication system is responsible of making data and information shared and available to all interacting entities. These entities can be machines such as AMI, substration, central data ware house, etc. or it could be human visualizing information in a terminal or a smart phone. This section discusses the communication system part of the smart grid.

From an architectural perspective, smart grid structure encompasses three high level layers. These levels are broken down as follows [6]

- Physical power and Control layer: It is responsible for the core functions such as generation, transmission and distribution of power.

- Communications layer: Functions as a bidirectional interface between utilities, consumers, grid components, and operators.
- Application layer: A provision of several applications as to consumers or control systems like Advanced Metering Infrastructure (AMI), Demand Response, Distributed Generation and Storage, Smart Charging of PHEVs and V2G Business and Customer Care, etc.

Figure 4 shows the overall architecture of SG, which can be decomposed into a power system layer and communications layer. The power system layer, is an integration of various electrical power generation systems, power transmission and distribution grids, substations, microgrids, customers, and control centers. Given the heterogeneity of the system, different technologies will be utilized in smart grid Communications layer [1]:

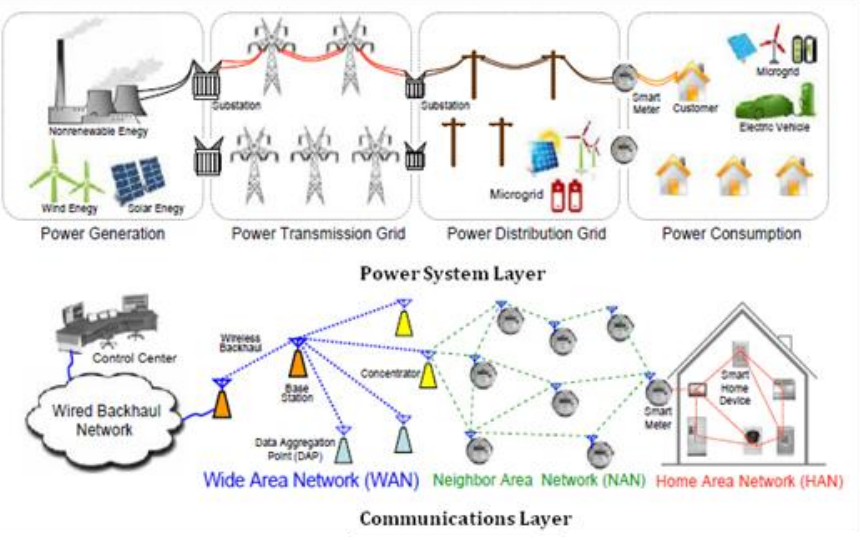

Figure 2: Communication Layer Vs Power system Layer [1]

- End user point holds customers premises that connect their devices with the home network of commercial power. In this part, Home HAN, Building BAN, or Industrial IAN Area Network exists in order to implement a group of devices that are equipped with smart grid components.

- Advanced Smart Infrastructure AMI demarcation starts with Neighborhood Area Network NAN/Field Area Network FAN points that connect meters to customers premises. AMI NAN/FAN from other side connects to the Core or WAN.

- Substation LANs are grouped in WAN where core/metro and backhaul demarcation are located. This point connects several devices like SCADA [7-8-9] (refers to Supervisory Control and Data Acquisition) inside a substation.

- LAN Network of AMI enterprises where control centre and data storage and analysis are located.

There are several important issues in communication network design, such as:

- Which communication technologies should be used to establish links between devices?

- Which network topologies are applicable in the context of electric grid infrastructures, and how communication technologies and grid geography affect the topology of the network?

- Which networking and transport protocols are the most appropriate for meeting the requirements of smart grid communications?

\section{SCTP Overview}

The Stream control transmission protocol stack and provides transport layer functions to many Internet applications. SCTP has been approved by the IETF as a proposed standard in 2000 and updated over the years. SCTP is a reliable transport protocol operating on top of a connectionless packet network such as IP[2]. SCTP protocol is a combination of TCP and 
UDP because each has some advantages and some drawback. UDP transmit the data in the form of stream and TCP transmit the data in the form of bytes. Transition of the data in the of stream is faster than transmission of bytes. SCTP (Stream Control Transmission Protocol) is a protocol for transmitting multiple streams of data at the same time between two end points that have established a connection in a network. Sometimes referred to as "next generation TCP" (Transmission Control Protocol) - or TCPng. SCTP is designed to make it easier to support a telephone connection over the Internet (and specifically to support the telephone system's Signaling System 7 - SS7 - on an Internet connection). A telephone connection requires that signaling information (which controls the connection) be sent along with voice and other data at the same time. SCTP also is intended to make it easier to manage connections over a wireless network and to manage the transmission of multimedia data. SCTP is a standard protocol (RFC 2960) developed by the Internet Engineering Task Force (IETF) [24]

\subsection{Multi-homing}

SCTP was designed to handle the signaling of telecommunications over IP[4]. Since telecommunications are very susceptible to time delays, every millisecond counts. Multi-homing enables systems that have multiple interfaces, for redundancy, to use one over the other without having to wait. Within SCTP one interface is established as the primary and the rest become secondary (figure 3). If the primary should fail for whatever reason, a secondary is selected and utilized. When the primary becomes available again, the communications can be transferred back without the application being aware there was an issue. While establishing the connections, the primary and secondary interfaces are checked and monitored using a heartbeat/heartbeat acknowledgement process that validates addresses, and maintains a Round Trip Time (RTT) calculation for each address. The RTT can indicate that the primary is slower than a secondary and allow for the communications to migrate to the secondary interface.

\subsection{Multi-streaming}

Using TCP, only one single data stream is allowed per connection [4]. All of the information must be passed through that one stream. SCTP allows multiple simultaneous data streams within a connection or association (figure 4). Each message sent to a data stream can have a different final destination, but each must maintain message boundaries. For example, systems cannot send parts of the same message through different streams; one message must go through one stream. When running an ordered data delivery system, if one of the packets is out of order or missing, the stream is blocked pending resolution to the order. This is called "Head-of-Line Blocking." With the use of multi-streams, only the stream that is affected would be blocked; the other streams would continue to flow.[24]

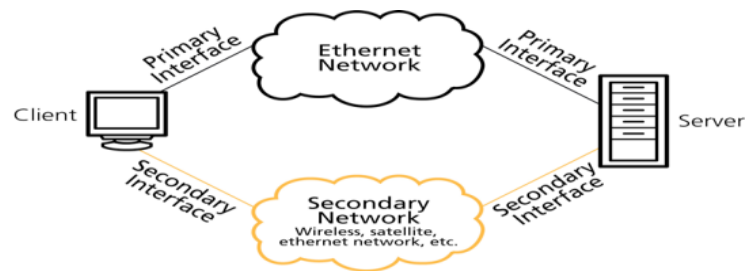

Figure 3 Example of Multi-homed Architecture.

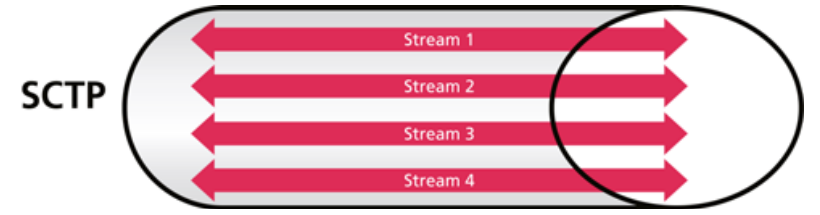

Figure 4 Multiple streams within a single association

\section{SCTP MULTI-HOMING FOR SMART GRID COMMUNICATIONS}

A general view of the system's network topology focuses on the Smart Energy home-area network communicating data back and forth to the consumer. In order to accomplish this two-way communication, certain access points and gateways are needed to relay statistical data between the end points. The Figure 5 depicts the general view of the proposed system.

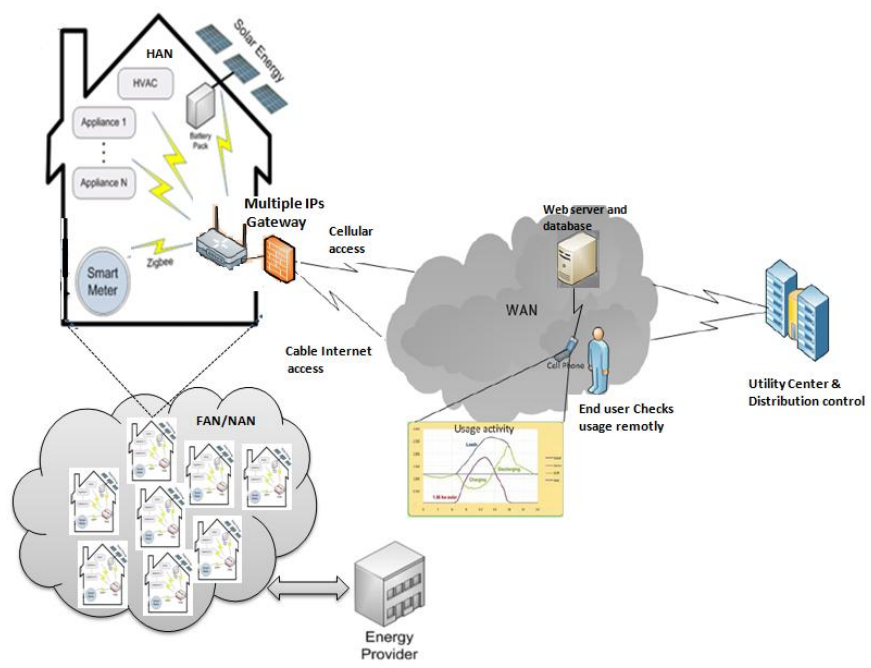

Figure 5 Multi-home in Smart Grid Communication Model

The home-area network relays consumption data between the energy providers via an advanced metering infrastructure. Consumer data is transported over the wan network (internet) to a web server via a gateway appliance, which represents a router which has multiple links connected to the other party. Each link has a public IP which is recommended in order to have different broadband connections access from other internet providers; it is advisable for a location to have different sources of internet access due to the possible issues that may arise with broadband access.

On the other hand we argue that by utilizing SCTP as the most likely transport protocol to function in smart grid communication, it can be possible to address the weaknesses that exist when using TCP. Since SCTP has such impressive features, one of which is Multi-homing, For example, if we consider Cable Internet in a complex of residences or office is disconnected from an internet providers side we will guarantee (at least temporarily) that another connection is available for smart grid service continuing since an independent source of the two internet accesses is provided. In the evaluation we depended on the instantaneous throughput of each packet leaving the gateway to the utility centre. Data exchange between appliances or devices and the other side of smart grid communication (utility centre) is conducted instantly even among indoor appliances. However, 
we consider only the activity of data exchange in-between the two end points. Throughput is a very common concept in networks, it is the actual received data that was sent at a specific time whether the data delivered is transferred in a wireless or wired medium. In the Results subsection, we will discuss in detail how our measurements were obtained for both throughput and delay for smart grid communications.

\section{SCTP MULTI-STREAMING FOR SMART GRID}

Multi-streaming is a feature in which SCTP derived its unique name and is described in more detail in Figure 4. Some measurements conducted of the Multi-streaming feature offered by SCTP. On the other hand, we also did some measurements of TCP which uses a stream of bytes in order to fragment data transmitting along to other peers. The TCP problem is the nature of the bytes stream that is sent unbroken into several streams; thus this is vulnerable to misleading bytes management. Actually the two TCP problems share creating the very well known TCP problem HoL blocking; the delayed packets waiting for other packets to be resembled will affect other unrelated ones. Thus, TCP is a byte-based stream protocol whom we were investigating against SCTP as a message-based, multi-streaming protocol. Due to the large predicted number of appliances and devices equipped with meters, smart grid communications will suffer utilizing TCP as its unique transport protocol. We will cover the delay effects resulted by HoL blocking against the one in SCTP and why it is wise to utilize SCTP as a prospective transmission (transport) protocol for smart grid communications.

\section{SIMULATION AND RESULTS}

In the simulation, is used ns-2, network simulator 2 (Version 2.35) [11] [28]. It is de most popular network simulator used by researchers. Ns-2 is an open source discrete event simulator used by the research community for in networking. This simulator provides support for simulation of TCP, routing a multicast protocols over wired and wireless (local and satellite) networks. [12-13-14]. In this work, we calculated data sent from the gateway along to the utility server of smart grid since we measured only an end-to-end (gateway to server) throughput during a handover to another link that takes place for both TCP and SCTP. Our multi homed scenario of smart grid communications simulation assumes that there are two independent broadband connections which connect a user location to the other party of the smart grid network. One is a Cable Internet access that represents a primary link with an upload of $1 \mathrm{Mgps}$ whereas the other one is a cellular $3 \mathrm{G}$ as a secondary link with an upload of 2Mgps; we used the default upload data rate of Cable Internet access, whereas for the $3 \mathrm{G}$ upload we assumed using $2 \mathrm{Mb}$ as an average of the uplink speed mentioned in 3GPP [13]

The simulation were conducted for one and three nodes scenario in order to test the effect of smart grid communications with variation in number of devices equipped with meters. All results are obtained from implementation of SCTP multi-homing and multi-streaming in the network simulator, and AWK scripts are used to extract required data from the results of simulations.

We scheduled 10 seconds as a simulation time and we have considered following performance metrics to conclude the effect of concurrent traffic, delay end to end (E2E) and throughput (total number of successful transmitted bits to the destination), when increasing the number of nodes to, we have noticed a considerable decrease of throughput due to the pressure applied on communications once a switchover with more traffic than the previous used for only a singular node. We can observe from the graph the effect of having many nodes on the throughput of SCTP that will impact on smart grid communications performance.

Figure 6 shows a comparison between the SCTP protocol with TCP in the case of three nodes and a switching from the primary link to the secondary one took place once the simulation time reached the second 4 for both of the transport protocols TCP and SCTP respectively. We observed the TCP throughput was degraded severely once the switchover was done in the time specified, against part the SCTP throughput remains stable and we can also observe that all SCTP and TCP traffic were almost similar until the handover to the secondary link (cellular connection) started receiving the traffic which shows a change in TCP behaviour to an unwanted degree although in this simulation the handover carries the traffic to a wider upload bandwidth. This note implies the concept that SCTP can manipulate several paths among peered points with better throughput.

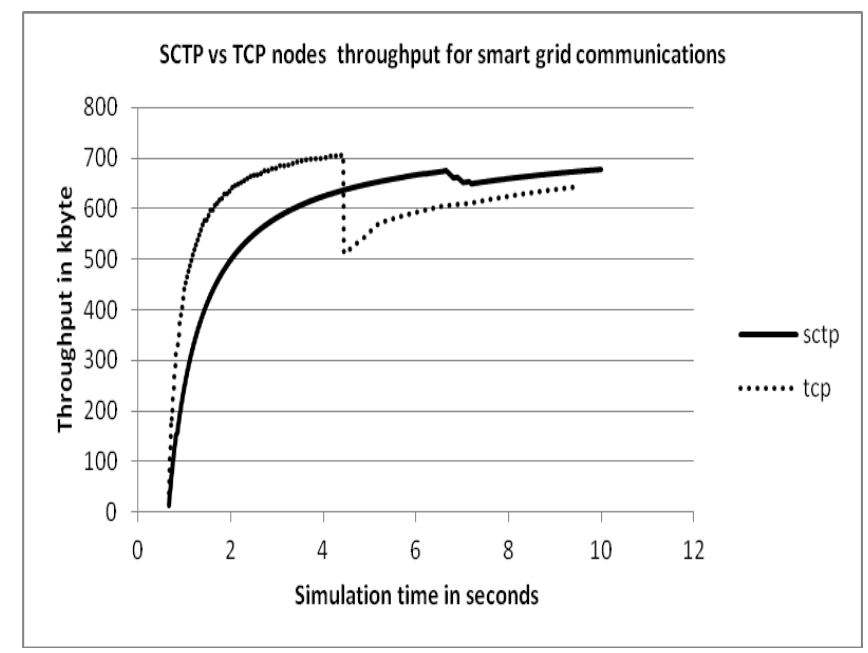

Figure 6: SCTP vs. TCP Three Nodes throughput in Multi-homed Connection of Smart Grid

In evaluation of delay is measured only an end-to-end (gateway gateway of HAN network to server) for both SCTP and TCP in an assumed single home smart grid communication environment. The delay calculation is assumed via measuring the instantaneous delay of every packet leaving the sender and targeting the receiver. As well, we did the overall delay calculation as a cumulative delay of all instantaneous ones up to the simulation end. The SCTP Multi-streaming feature was enabled whereas in TCP we have only one option to measure the delay which is via utilizing the single byte stream method. In figure 7 we can notice the delay difference between SCTP and TCP in terms of performance optimization, in this figure we find that the delay SCTP reached 0.27 seconds and then was recovered to its normal delay time then we calculated the TCP delay for the same end to end topology. In the second 3.7 (as SCTP trial was indicated) the delay gets raised to a value that was over the SCTP trial. This value is 0.4 seconds, which was 0.33 second more than SCTP side, this is demonstrates the delay effect that is reflected in a single homed end-to-end network of smart grid communications that operates using TCP as its transport protocol. 


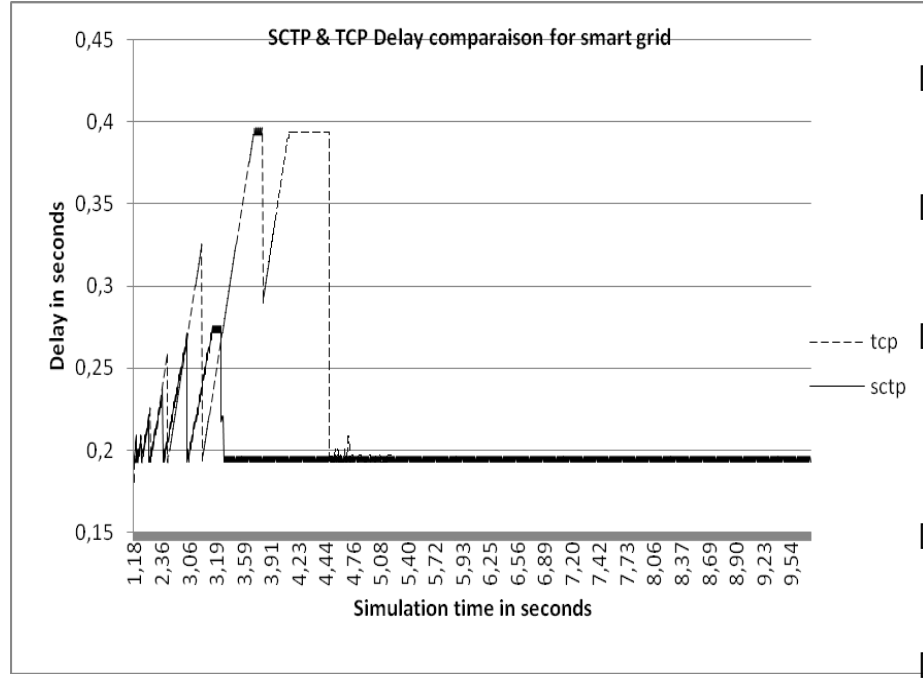

Figure 7: SCTP and TCP Delays comparison for Smart Grid Network

\section{CONCLUSION AND FUTURE WORK}

In this paper is presented an introduction to smart grid and smart grid communication network. An overview of SCTP multi-homing and multi-streaming characteristics is also outlined. The main work presented is to propose a mechanism of enhancing smart grid communications performance by using SCTP protocol instead of TCP.

The results presented are built on two major measurements which are delay and throughput for both SCTP and TCP of smart grid communications and show a moderate benefit from using SCTP due to superiority evidence of SCTP over TCP. in future work, sctp protocol can be applied in many field such as heterogeneous network of wired and wireless/mobile nodes while maintaining quality and reliability of service availability when switching between sources exists in situations where a renewable energy (e.g., solar or wind) fed small cell base station (green cellular) is used as an alternative and assistant to a traditional energy fed base station.

\section{REFERENCES}

[1] Mohammad S. Obaidat, Alagan Anpalagan, and Isaac Woungang "Green Information and Communication Systems", Handbook, ISBN: 978-0-1241-5844-3, First edition 2013 Elsevier Inc

[2] António Grilo, Helena Sarmento, Mário Nunes, José Gonçalves, Paulo Pereira, Augusto Casaca, Carlos Fortunato, «A Wireless Sensors Suite for Smart Grid Applications », Information Technology for Energy Applications 2012, Lisbon, Portugal, September 6-7, 2012.

[3] R. J. Robles and T. Kim "Applications, Systems and Methods in Smart

Home Technology: AReview", International Journal of Advanced Science and TechnologyVol. 15, February, 2010

[4] A dithya Shreyas, "analysis of communication protocols for neighborhood area network for smart grid" Master of computer engineering, 2010

[5] National Institute of Standards and Technology, US Dept of Commerce, "NIST Framework and Roadman for Smart Grid Interoperability
Standards release 1.0", [www.nist.gov]

[6] D. Leeds, A white paper in, The Smart Grid in 2010: Market Segments, Applications and Industry Players, July 2009.

[7] T. Lewis, Critical Infrastructure Protection in Homeland Security: Defending a Networked Nation, April 2006, Wiley and Sons 486 pages

[8] Emilo Ancillotti, Raffaele Bruno, Marco Conti, "the role of communication systems in smart grids: architectures, technical solutions and research challenges", Computer Communications, vol 36, issues 17-18, November, December 2013, P. 1665- 1697]

[9] Wenye Wang, Yi Xu, Mohit Khanna, "A survy on the communication architectures in smart grid", Computer Netwrks 55 (2011) 3604-3629]

[10] R. Stewart, Q. Xie, K. Morneault, C. Sharp, H. Schwarzbauer, T. Taylor,I. Rytina, M. Kalla, L. Zhang, and V. Paxson. "Stream control transmission

Protocol'. RFC 2960, Oct. '00

[11] The Network Simulator Project $-\mathrm{ns}-2$ (release 2.35).[online] available in URL: http://www.isi.edu/nsnam/

[12] Nico Saputro, Kamal Akkaya, Suleman Uiudag, “ A survey of routing protocols for smart grid communications", Computer Networks, 56 (2012) 2742 2771

[13] The 3rd Generation Partnership Project (3GPP), HighSpeed Packet Access (HSPA), 2012.

[14] Adrian Clark and Christopher J. Pavlovski, "Wireless Networks for the Smart Energy Grid: Application Aware Networks", International MultiConference of Engineers and Sciebtists 2010 volII, March 17 - 19 2010, Hong Kong

[15] Erwin P. Rathgeb "On SCTP multihoming performance", Telecommunication Systems, Vol. 31, No 2-3, 2006, pp. 141-161, 2006. DOI: 10.1007/s11235-006-6517-7

[16] Rahul Amin, Jim Martin, Xuehai Zhou, "Smart Grid Communication using Next Generation Heterogeneous Wireless Networks “, IEEE Third International Conference on Smart Grid Communications, Tainan, Taiwan 5 - 8 November 2012, pp. 229-234

[17] R. J. Robles and T. Kim "Applications, Systems and Methods in Smart Home Technology: AReview", International Journal of Advanced Science and TechnologyVol. 15, February, 2010

[18] Yang-Wen Chen, Arak Sae Yuan, Kuan-Ta Lu and Quincy Wu, "The Study of 6LoWPAN with Multihoming in Smart Grid", Seven International Conference on Sensing Technology, 2013.

[19] Q.-Dung Ho, Yue Gao, and Tho Le-Ngo, “ Challenges and Research Opportunites in Wireless Communication Networks for Smart Grid", IEEE Wirelesss Communication June 2013.

[20] V. C. Gungor and F. C. Lambert, "A survey on communication networks for electric system automation," Comput. Netw., vol. 50, no. 7, pp. $877-$ 897, May 2006. 
[21] Jen-Yi Pan,Min-Chin Chen, Ping-Cheng Lin, and KuoLun Lu, Quality-Aware SCTP inWireless Networks" EURASIP Journal onWireless Communications and Networking Volume 2010, Article ID 820578, 14 pages

[22] S. Fu and M. Atiquzzaman, SCTP: State of the Art in Research,Products, and Technical Challenges, IEEE Communications Magazine, April 2004.
[23] R. Stewart and P. Amer, Why is SCTP needed given TCP and UDP are widely available?, In Internet Society, 10 September 2007.

[24] Vandana Vijay Belkhode, "UDP-Based Multi-Stream Communication Protocol Using NS2", International Journal on Recent and Innovation Trends in Computing and Communication, Volume: 2 Issue: 3, Mars 2014. 